\title{
An audit of prescribing for type 2 diabetes in primary care: optimising the role of the community pharmacist in the primary healthcare team
}

\author{
Michael J. Twigg ${ }^{1}$, James A. Desborough ${ }^{2}$, Debi Bhattacharya ${ }^{2}$ and David J. Wright ${ }^{3}$ \\ ${ }^{1}$ PhD Student, Medicines Management Research Team, School of Pharmacy, University of East Anglia, Norwich, UK \\ ${ }^{2}$ Lecturer, Medicines Management Research Team, School of Pharmacy, University of East Anglia, Norwich, UK \\ ${ }^{3}$ Professor, Medicines Management Research Team, School of Pharmacy, University of East Anglia, Norwich, UK
}

\begin{abstract}
The Government has identified that the pharmacist should have greater involvement in the management of long-term conditions. The aim of this audit was to determine the adherence to National Institute for Health and Clinical Excellence guidelines for type 2 diabetes patients and identify whether there is a potential role for pharmacists in their long-term management. All prescribing, in 194 patients, was within guidance for anti-hyperglycaemics. In all, $87.4 \%$ of patients prescribed an anti-hypertensive were prescribed an angiotensin-converting enzyme inhibitor or equivalent. A large number of patients remain uncontrolled with respect to blood glucose or blood pressure. There are four potential reasons for this: patients require additional therapy; current therapy has not been optimised; current therapy is not working; or the patient is not fully adherent. Therefore, there may be a role for the pharmacist either in therapy optimisation or improving patient adherence to current therapy in order to support more patients reaching national targets.
\end{abstract}

Key words: community pharmacist; prescribing; type 2 diabetes

Received 1 February 2012; revised 8 May 2012; accepted 28 May 2012;

first published online 13 July 2012

\section{Introduction}

In 2007/2008, the National Health Service (NHS) was treating $\sim 2.1$ million people with diabetes mellitus (The Health and Social Care Information Centre, 2008) at significant cost to the NHS. The majority of patients $(85 \%)$ are diagnosed with type 2 diabetes, which is largely controlled by oral medication. The total cost of oral anti-diabetic drugs issued in primary care to the NHS in the year September 2008 was £161.3 million, representing a $10 \%$ increase in the number of medicines prescribed (The Health and Social Care Information

Correspondence to: Michael J. Twigg, Medicines Management Research Team, School of Pharmacy, University of East Anglia, Norwich NR4 7TJ, UK. Email: m.twigg@uea.ac.uk
Centre \& The Yorkshire and Humber Public Health Observatory, June 2009). Diabetes is a chronic condition that, if not treated properly, can lead to complications such as retinopathy, nephropathy and neuropathy, which can further increase costs to the NHS.

The National Institute for Health and Clinical Excellence (NICE) has published guidance on prescribing for type 2 diabetes (NICE, 2009) to which prescribers are encouraged to adhere in order that patients receive the most cost-effective care. Within primary care, the management of patients with diabetes has become a primary responsibility for the practice nurse with a specialisation with support from the patient's doctor. Other professions within the primary care team have defined roles in the care of patients, for 
example, the dietician and the podiatrist. The community pharmacist, part of the wider primary care team, will see the patient on a regular, monthly basis when they collect their medicines but does not have a defined role. Community pharmacists have the opportunity to develop a long-term relationship with these patients and those not attending the medical practice to potentially improve the use of the medicines, the condition and resulting co-morbidities.

The audit determined whether prescribing was in accordance with NICE guidance; patients were adequately controlled and identified any areas where role of the community pharmacist in the primary healthcare team could be optimised.

\section{Method}

Approval for the audit was sought from NHS Norfolk's Research and Development department. NHS Norfolk's prescribing advisors identified 10 practices as potential participants in the audit based on their likelihood of participation. These practices represented a spread in total anti-diabetic spending per head of population in NHS Norfolk and a spread of scores on the Quality and Outcomes Framework (QOF) indicator DM5 (number of patients who had received an $\mathrm{HbA}_{1 \mathrm{C}}$ blood test within the previous 15 months).

Each practice was approached by the relevant prescribing advisor and asked whether they would like to participate. Once agreed, an alphabetical list of all patients at each practice, who met the following inclusion criteria, was obtained from the practice manager for the researcher.

\section{Inclusion criteria}

- Confirmed diagnosis of type 2 diabetes within the previous five years

- Aged over 18 years

- Not prescribed insulin

A random number generator was used to select 25 patients from the alphabetical list provided. If there were fewer than 25 patients eligible, then all patients were used. Once a patient was selected, the date of diagnosis, age and medication prescribed was checked to ensure that the patient met the inclusion criteria.

\section{Audit standards}

$100 \%$ adherence to NICE guidance for the management of type 2 diabetes in terms of the following:

- Clinical monitoring intervals and achievement of target levels were provided by NICE for $\mathrm{HbA}_{1 \mathrm{C}}$, blood pressure (BP), weight, cardiovascular $(\mathrm{CV})$ risk, renal function, lipids, retinal and neurological examinations.

- Prescribed therapy in accordance with NICE stepwise management approach for diabetes and co-morbid conditions covered by Clinical Guideline 87 (NICE, 2009).

For the clinical tests recommended by NICE to be conducted every six months, records were reviewed to determine whether the patient had received the appropriate number of tests in the previous 12 months. A two-week allowance for non-attendance at the practice was incorporated. Data were not collected to allow the calculation of renal function.

Medication prescribed for the treatment of diabetes was recorded along with the sequence in which it was initiated and whether there was a clinical need for that medicine. Information obtained on BP medication was used to determine whether a patient with hypertension and diabetes was prescribed an angiotensin-converting enzyme (ACE) inhibitor or angiotensin II receptor antagonist (AIIRA).

The audit tool was tested before data collection by one of the practice managers to determine whether it was possible to collect all the data the research team set out to collect. This information was fed back to the research team so that alterations to the tool could be made.

\section{Results}

Nine practices agreed to participate in the audit from which data for 194 patients were collected. The mean (SD) age in years for patients was 65.13 (12.1) and the mean (SD) length of time since diagnosis was 29 (17.4) months. Table 1 describes the variation in demographics of the practices sampled. The team achieved a good distribution in terms of practice position in terms of $\mathrm{QOF}$ indicator DM5, dispensing/non-dispensing and a mixture of rural, town and city practices. 
Table 1 Practice demographics (QOF 2009-2010 data)

\begin{tabular}{lllll}
\hline Practice & $n$ & $\begin{array}{l}\text { No. of patients on the diabetes } \\
\text { register (\% of practice population) }\end{array}$ & $\begin{array}{l}\text { QOF indicator DM5 } \\
\text { result (centile) }\end{array}$ & $\begin{array}{l}\text { Dispensing/ } \\
\text { non-dispensing }\end{array}$ \\
\hline 1 & 25 & $269(4.9)$ & 68 & Docation \\
2 & 22 & $336(4.2)$ & 73 & Dispensing \\
3 & 25 & $261(3.7)$ & 85 & Dispensing \\
4 & 24 & $133(4.3)$ & 76 & Nispensing \\
5 & 13 & $171(3.0)$ & 42 & Non-dispensing \\
6 & 25 & $178(4.2)$ & 68 & City \\
7 & 10 & $300(4.1)$ & 42 & Dispensing \\
8 & 25 & $261(4.0)$ & 75 & Dispensing \\
9 & 25 & $403(4.1)$ & 29 & Non-dispensing \\
\hline
\end{tabular}

QOF $=$ Quality and Outcomes Framework.

Table 2 Adherence to testing criteria ( $n=194$ for all tests)

\begin{tabular}{lll}
\hline NICE criterion & $\begin{array}{l}\text { Recommended frequency } \\
\text { of test (months) }\end{array}$ & $\begin{array}{l}\text { \% Adherence to monitoring } \\
\text { frequency (95\% Cl) }\end{array}$ \\
\hline HbA $_{1 \mathrm{C}}$ measurement & 6 & $79.4(73.7-85.1)$ \\
$\mathrm{BP}$ measurement & 6 & $71.6(65.3-77.9)$ \\
Weight and BMI measurement & 12 & $92.3(88.5-96.1)$ \\
Cardiovascular risk assessment & 12 & $31.4(24.9-37.9)$ \\
Lipid measurements & 12 & $95.4(92.5-98.3)$ \\
Renal function & 12 & $93.3(89.8-96.8)$ \\
\hline
\end{tabular}

NICE = National Institute for Health and Clinical Excellence; $\mathrm{BP}=$ blood pressure; $\mathrm{BMI}=$ body mass index.

Table 3 Patient outcomes

\begin{tabular}{lll}
\hline Clinical test & $\begin{array}{l}\text { Number of patients } \\
\text { with recorded value }\end{array}$ & $\begin{array}{l}\text { Adherence \% } \\
(95 \% \mathrm{Cl})\end{array}$ \\
\hline $\mathrm{HbA}_{1 \mathrm{C}}<58 \mathrm{mmol} / \mathrm{mol}(7.5 \%)$ & 194 & $82.4(77.0-87.8)$ \\
$\mathrm{Blood}$ pressure $<140 / 80 \mathrm{mmHg}$ & 194 & $61.3(54.3-68.3)$ \\
Lipids $<4.0 \mathrm{mmol} / \mathrm{L}$ & 194 & $47.4(40.3-54.5)$ \\
$\mathrm{BMI}$ & 186 & $14.0(9.0-19.0)$ \\
$\quad$ Underweight/ideal weight & & $28.0(21.5-34.5)$ \\
$\quad$ Overweight & & $31.2(24.5-37.9)$ \\
Obese & 29 & $26.9(20.5-33.3)$ \\
$\quad$ Morbidly obese & & $6.9(-2.3-16.1)$ \\
Cardiovascular risk assessment (\%) & & $24.1(8.5-39.7)$ \\
$\quad<10$ & & $69.0(52.2-85.8)$ \\
$\quad 10-20$ & & \\
$>20$ & & \\
\hline
\end{tabular}

$\mathrm{BMI}=$ body mass index

Table 2 summarises adherence to the audit standards for the clinical tests and the number of patients achieving targets for those parameters. Practices performed well for the number of patients with a recorded weight, lipid and renal function; however, for $\mathrm{HbA}_{1 \mathrm{C}}$ and $\mathrm{BP}$ practices failed to achieve near the $100 \%$ adherence standard set by this audit. The breakdown of clinical results, CV risk and body mass index is shown in Table 3, which demonstrates the large number of patients that remain uncontrolled with their diabetes especially with respect to BP and cholesterol. 


\section{Medication}

There were 139 (71.6\%) patients who were prescribed at least one medicine to manage their diabetes, 38 (19.6\%) patients were prescribed two medicines and four $(2.1 \%)$ patients were treated with three oral anti-hyperglycaemics. Fifty-five $(28.4 \%)$ patients were not prescribed any medicines for their diabetes and were presumed to be controlled by diet alone of which only three of these patients were classed as uncontrolled. All first-, second- and third-line therapies matched NICE recommendations. In 36 out of the 38 patients who were prescribed second-line therapy, their $\mathrm{HbA}_{1 \mathrm{C}}$ level was sufficiently high at the time of initiation $(>6.5 \%)$ to warrant that addition. For third-line therapy, three out of four patients met the NICE recommendations for additional therapy $\left(\mathrm{HbA}_{1 \mathrm{C}}>7.5 \%\right)$.

For those prescribed a thiazolidinedione or dipeptidyl-peptidase- 4 inhibitor, NICE states that there must have been a $0.5 \%$ drop in their $\mathrm{HbA}_{1 \mathrm{C}}$ level after six months of treatment for it to be continued. This was the case in four out of the twelve prescribing incidences. Two did not see a decrease, of which one was stopped and one was not. Data were unavailable for the remaining patients as they had only been recently prescribed.

A total of 135 patients were prescribed at least one medicine for hypertension of which 118 (87.4\%) were prescribed an ACE inhibitor or an AIIRA. There were $37(27.4 \%)$ patients prescribed a $\beta$-blocker, currently 4 th line in NICE guidance, and are not recommended because of their effect on carbohydrate metabolism.

Finally, $74.7 \%$ of patients were prescribed a lipid-lowering drug and $39.7 \%$ of patients were prescribed an anti-thrombotic drug, for example, aspirin. Of patients who had uncontrolled lipid levels (total cholesterol level $>4.0 \mathrm{mmol} / \mathrm{L} ; n=101$ ), $38.6 \%$ were not prescribed any lipid-lowering medication.

\section{Discussion}

From the results, it can be seen that there is generally good adherence to the audit standards with reference to NICE guidance. The majority of patients were undergoing the appropriate clinical tests at the recommended intervals. The medicines for diabetes and BP are being prescribed in accordance with recommendations made by NICE. Medication to treat hypertension was simply listed on the audit tool and not sequenced as with diabetes medication. This was because of the complex nature of a patient's condition and that some of the medicines had been prescribed pre-diabetes diagnosis, therefore making it difficult to compare with the NICE guidance. This finding has been confirmed in previous research (Simoens et al., 2009). However, at the point at which the audit was conducted the majority of patients were prescribed first-line recommended ACE inhibitors or equivalent. This is ideally the first-line therapy for patients with type 2 diabetes because of its renoprotective effects. The low prescribing rate of aspirin maybe a reflection of the recent evidence that has been made available regarding the limited benefit of aspirin for primary prevention of cardiovascular events in diabetes (De Berardis et al., 2009; Chunyu et al., 2010).

A large proportion of patients did not receive a blood glucose or BP measurement at least every six months or a CV risk assessment every 12 months. $\mathrm{BP}$ monitoring is a service widely available within community pharmacies and therefore, with appropriate communication systems between practices and pharmacies, may be a means for pharmacy to contribute to adherence to NICE guidance. The reporting of $\mathrm{CV}$ risk assessments varies according to practice, and therefore further research would be warranted to determine the exact figure. The majority of data required to perform a $\mathrm{CV}$ risk assessment was found in patient records; this deficit in reporting could be rectified by the appropriate use of software within the practice.

These results indicate that there are still a number of patients who are not controlled on their current medications with reference to $\mathrm{HbA}_{1 \mathrm{C}}, \mathrm{BP}$ and cholesterol. This may be due to inadequate dose titration by the prescriber (data that were not collected), the need for additional medicines to be prescribed, current medicines not working or the patient being non-adherent and the pharmacist may have a role in addressing each one of these.

Through regular attendance at the pharmacy to collect their medicines, it may be appropriate for the community pharmacist to become more involved with these patients. In response to a significant lack of therapy optimisation, through patients not taking their medicines, the Government has introduced 
brief pharmacist-led interventions, for example, medicine use reviews and the new medicine service, to increase the responsibility placed on the pharmacist in patients with long-term conditions. However, these new services do not have a robust evidence base supporting their implementation. This audit set out to determine whether the community pharmacist could contribute to the health of the patient. It appears that pharmacists should be focusing on patient adherence or medicine optimisation rather than reviewing prescriber's adherence to NICE guidance.

\section{Limitations}

This audit was only conducted in nine practices in Norfolk, and therefore the results have limited generalisability. The audit covered the medicines prescribed for diabetes and co-morbid conditions. It did not include other aspects of care covered by the NICE guidance such as smoking status or lifestyle advice given to the patient. Half the practices audited were dispensing practices and as such patients will not have access to a community pharmacist and ways of building a model for these patients need to be considered.

\section{Conclusion}

Prescribing for type 2 diabetes is generally in line with national guidance. There are, however, still uncontrolled patients and this may be due to a lack of dose optimisation, the need for further medicines to control their condition or adherence problems on the part of the patient. The community pharmacist, who sees the patient on a monthly basis when they collect their prescription, could have a role in improving dose optimisation and adherence in uncontrolled patients. In future, they may be able to identify these patients by the use of summary care records that are being phased in across the country. However, for this to be effective, good lines of communication between themselves and the medical practices would be required.

\section{Acknowledgements}

The authors thank Simone Johnson for piloting this audit tool on behalf of the research team and all the practice managers for allowing the research team in to conduct the research. They also thank the prescribing advisors at NHS Norfolk who arranged an introduction to all the practices.

\section{References}

Chunyu, Z., Aijun, S., Peng, Z., Chaoneng, W., Shuning, Z., Mingqiang, F., Keqiang, W., Yunzeng, Z. and Junbo, G. 2010: Aspirin for primary prevention of cardiovascular events in patients with diabetes: a meta-analysis. Diabetes Research and Clinical Practice 87, 211-18.

De Berardis, G., Sacco, M., Strippoli, G., Pellegrini, F., Graziano, G., Tognoni, G. and Nicolucci, A. 2009: Aspirin for primary prevention of cardiovascular events in people with diabetes: meta-analysis of randomised controlled trials. British Medical Journal 339, b4531.

National Institute for Health and Clinical Excellence (NICE) 2009: Type 2 diabetes: the management of type 2 diabetes. Clinical guideline 87. London: National Institute of Health and Clinical Excellence.

Simoens, S., De Coster, S., Dethier, M., Lenie, J., Meuwissen, P. and Laekeman, G. 2009: Cardiovascular drug use prior to diagnosis of diabetes: a pilot study in community pharmacy. International Journal of Pharmacy Practice 17, 127-29.

The Health and Social Care Information Centre. 2008: QOF disease prevalence 2007/8. Retrieved 11 August 2010 from http://www.ic.nhs.uk

The Health and Social Care Information Centre\& The Yorkshire and Humber Public Health Observatory. June 2009: Prescribing for diabetes in England - an update: 2002-2008 Retrieved August 2010 from http://www.ic. nhs.uk. 